\title{
Essais
}

Revue interdisciplinaire d'Humanités

Hors-série 6 | 2021

Agrobiodiversité et territoires

\section{Valorisation des races animales locales par des produits liés à leur territoire : cinq leçons tirées de cas variés en France}

Valuing local animal breeds through products linked to their territory: five lessons from various cases in France

Étienne Verrier, Lucie Markey et Anne Lauvie

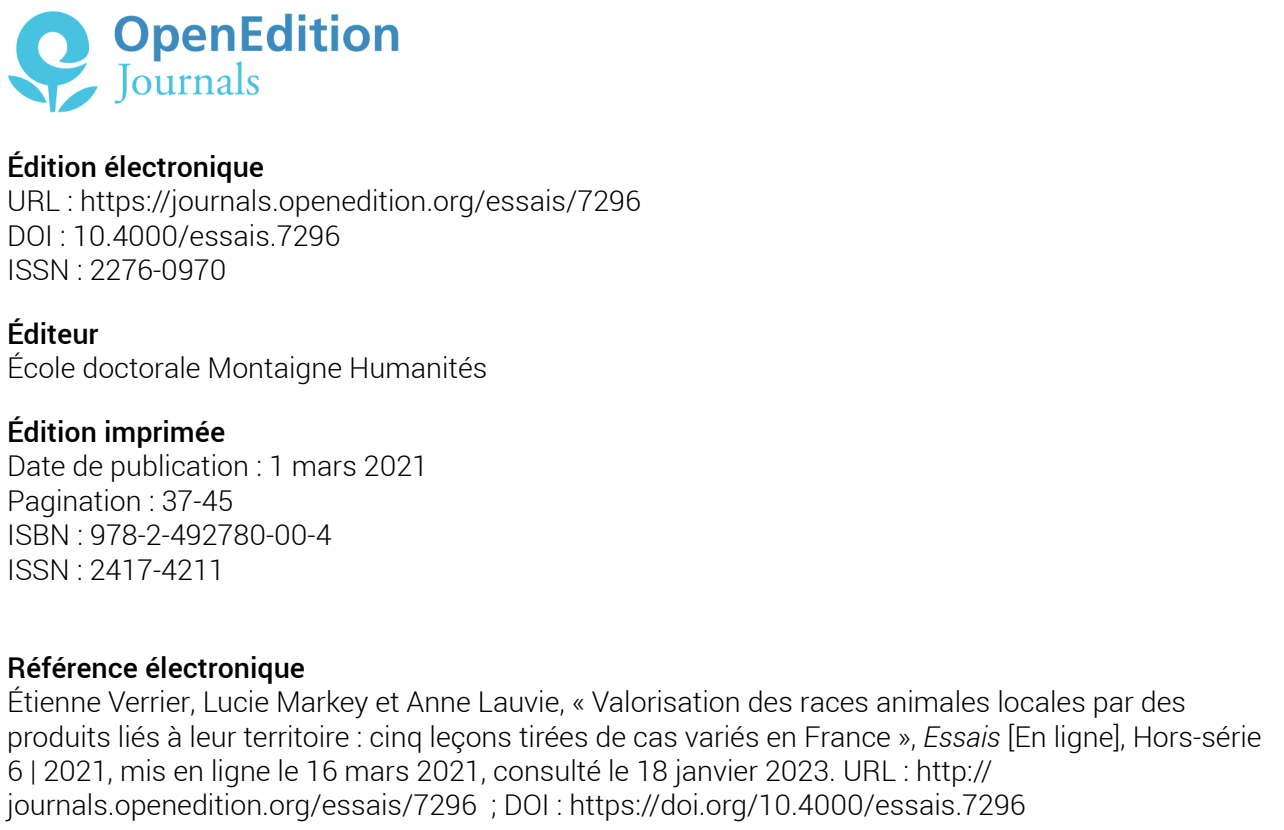

Tous droits réservés 


\section{Valorisation des races animales locales par des produits liés à leur territoire : cinq leçons tirées de cas variés en France}

\section{Étienne Verrier, Lucie Markey, Anne Lauvie}

La biodiversité animale domestique est à la fois une résultante de l'activité d'élevage et une ressource pour celle-ci ${ }^{1}$. Apparue aux XVIII ${ }^{e}$ et $\mathrm{XIX}^{\mathrm{e}}$ siècles en Europe, la notion de race animale demeure opérationnelle dans la plupart des situations de gestion collective de cette biodiversité domestique. Cette notion ne représente pas une subdivision naturelle d'une espèce donnée mais constitue le résultat d'un projet humain. De ce fait, elle comprend plusieurs dimensions : biologique, sociale, administrative, économique, liée à des systèmes et des pratiques, et culturelle ${ }^{2}$. En France, comme dans de nombreux autres pays, une race est qualifiée de locale si elle est « majoritairement liée à un territoire donné, par ses origines, son lieu et son mode d'élevage " ${ }^{3}$.

Depuis la seconde moitié du XXe siècle, la biodiversité domestique animale est menacée, notamment car de nombreuses races locales ont été remplacées par quelques races spécialisées et plus productives ${ }^{4}$. La préservation de ces races locales, voire leur relance, dépend alors de plusieurs conditions, dont la première se révèle toujours essentielle : (i) la motivation des éleveurs et leur cohésion, (ii) une adaptation de la race à des conditions de milieu et des systèmes d'élevage spécifiques, (iii) la fourniture de produits apportant un revenu satisfaisant aux éleveurs, et (iv) la fourniture de services autres qu'ali-

1 Xavier Rognon, Denis Laloë, Emmanuelle Vila, Étienne Verrier, La domestication et la constitution des ressources génétiques, in Étienne Verrier, Denis Milan, Claire Rogel-Gaillard (Éds), Génétique des animaux d'élevage, Quae Éditions, 2020, p. 15-26.

2 Annick Audiot, Races d'hier pour l'élevage de demain, INRA Éditions, Paris, 1995, 229 p.

3 Code Rural et de la Pêche, Article D653-9.

4 Annick Audiot, Races d'hier pour l'élevage de demain, op. cit. 
mentaires ${ }^{5},{ }^{6}, 7,{ }^{7},{ }^{9}$. L'objet de cet article est d'examiner la diversité qui existe en France des formes de valorisation des races locales via des produits liés à leur territoire (condition iii ci-dessus) et de tirer des enseignements de divers cas étudiés de près.

\section{La grande diversité des initiatives de valorisation des races locales via leurs produits}

Il est difficile de dresser un inventaire exhaustif et actualisé des multiples initiatives prises en faveur de la valorisation de races locales. On peut toutefois donner des grandes lignes et fournir quelques exemples. Au titre des grandes lignes, on peut mettre en avant la diversité (Tableau 1) : diversité des types de produits, de la dimension individuelle ou collective des initiatives et des outils mobilisés. Cette diversité n’a toutefois pas le même visage sur tout le territoire national.

De façon schématique, au sud d'une ligne Strasbourg-Bayonne, les initiatives sont très majoritairement collectives et mobilisent des signes officiels (SIQO), le plus souvent l'appellation d'origine protégée (AOP). C'est ainsi le cas des deux races bovines locales des Alpes du nord, des trois races ovines laitières des Pyrénées, de la race ovine Corse et de la race caprine Rove, dont l'utilisation figure explicitement dans le cahier des charges des AOP fromagères correspondantes. C'est aussi le cas de nombreuses races locales valorisées par des produits carnés labellisés (chez les bovins, les ovins, le porc ou le poulet). Dans ce groupe, notons le cas très original du Porc Basque dont la valorisation a démarré par l'initiative et l'engagement d'une entreprise privée de salaison et s'est progressivement muée en une démarche collective, l'adhésion des éleveurs aidant, avec dépôt d'une marque collective puis obtention d'une AOP.

Au nord de cette ligne Strasbourg-Bayonne, il existe bien des démarches collectives avec SIQO, mais les initiatives sont plus multiformes. Notamment, dans le cas de plusieurs races bovines (Bretonne Pie Noir, Bazadaise, Parthenaise),

5 Annick Audiot, Races d'hier pour l'élevage de demain, op. cit.

6 Étienne Verrier, Michèle Tixier-Boichard, Roland Bernigaud, Michel Naves, Conservation and values of local livestock breeds: usefulness of niche products andlor adaptation to specific environments, Animal Genetic Resources Information 36, 2005, p. 21-31.

7 Anne Lauvie, Annick Audiot, Nathalie Couix, François Casabianca, Hélène Brives, Étienne Verrier, Diversity of rare breed management programs: between conservation and development, Livestock Science 140, 2011, p. 161-170.

8 Anne Lauvie, Nathalie Couix, Étienne Verrier, No development, no conservation: lessons from the conservation of farm animal genetic resources, Society and Natural Resources, 27, 2014, p. 13311338.

9 Jean-Baptiste Borrès, Claude Béranger, Joseph Bonnemaire, Philippe Lacombe, JeanLouis Rouquette, L'Aubrac, 50 ans de développement, Éditions de l'Aube, PNR de l'Aubrac, 2019, p. 107-153. 
la valorisation passe essentiellement par le cumul d'initiatives individuelles d'éleveurs : transformation du lait à la ferme, vente directe, découpe à la ferme et caissettes de viande, circuits courts avec des bouchers locaux, etc. Notons que cette situation n'est pas incompatible avec une démarche collective pour un produit ciblé, comme c'est le cas en race Bretonne Pie Noir avec le Gwell (un lait fermenté) ${ }^{10}$.

Tableau 1 : Exemples de races locales dont les produits sont valorisés d'une façon spécifique, selon le type d'initiative et l'outil mobilisé. Types de produit : $\mathrm{F}=$ fromages ; $\mathrm{L}=$ autres produits laitiers ; $\mathrm{M}=$ miel $; \mathrm{s}=$ salaisons $; \mathrm{v}=$ viande. Types d'initiative $: \mathrm{c}=$ collective $; \mathrm{E}=\mathrm{d}$ 'entreprise ; I = individuelles. Outils mobilisés : sIQO = signe d'identification de la qualité et de l'origine ; AOP = appellation d'origine protégée ; IGP = indication géographique protégée ; LR = label rouge.

\begin{tabular}{|c|c|c|c|c|c|}
\hline \multirow{2}{*}{ Espèce } & \multirow{2}{*}{ Race } & \multirow{2}{*}{$\begin{array}{c}\text { Type } \\
\text { de produit }\end{array}$} & \multirow{2}{*}{$\begin{array}{c}\text { Type } \\
\text { d'initiative }\end{array}$} & \multicolumn{2}{|c|}{ Outil mobilisé } \\
\hline & & & & SIQO & $\begin{array}{l}\text { Marque } \\
\text { collective }\end{array}$ \\
\hline \multirow[t]{7}{*}{ Bovins } & Abondance & $\mathrm{F}$ & C & AOP & \\
\hline & Tarentaise & $\mathrm{F}$ & $\mathrm{C}$ & AOP & \\
\hline & Bretonne Pie Noir & $\mathrm{F}, \mathrm{V} / \mathrm{L}$ & $\mathrm{I} / \mathrm{C}$ & & non / oui \\
\hline & Rouge des Prés & v & $\mathrm{C}$ & AOP & \\
\hline & Parthenaise & $\mathrm{v}$ & I & & \\
\hline & Bazadaise & $\mathrm{v}$ & I & & \\
\hline & Aubrac & $\mathrm{v}$ & $\mathrm{C}$ & IGP, LR & \\
\hline \multirow[t]{3}{*}{ Ovins } & Races lait. Pyrénées & F & $\mathrm{C}$ & AOP & \\
\hline & Barégeoise & $\mathrm{v}$ & $\mathrm{C}$ & AOP & \\
\hline & Corse & $\mathrm{F}$ & $\mathrm{C}$ & AOP & \\
\hline Caprins & Rove & $\mathrm{F}$ & $\mathrm{I}$ & AOP & \\
\hline \multirow[t]{2}{*}{ Porc } & Basque & $s$ & E, C & AOP & oui \\
\hline & Nustrale & $s$ & $\mathrm{C}$ & AOP & \\
\hline \multirow[t]{3}{*}{ Poulet } & Gauloise Bresse $\mathrm{Bl}^{\text {che }}$ & $\mathrm{v}$ & $\mathrm{C}$ & AOP & \\
\hline & Coucou de Rennes & $\mathrm{v}$ & C & & \\
\hline & Gasconne & $\mathrm{v}$ & $\mathrm{C}$ & & oui \\
\hline Abeille & Écotype Corse & M & $\mathrm{C}$ & AOP & \\
\hline
\end{tabular}

\section{Le marché, l'offre et la demande}

Un objectif majeur des initiatives de valorisation des races locales au travers de leurs produits est d'obtenir pour les éleveurs des prix sensiblement plus élevés que la moyenne, afin de compenser la productivité généralement modérée de ces races et les éventuelles contraintes de leur milieu d'élevage. C'est typiquement ce que l'on observe depuis longtemps pour les AOP fromagères

10 Clémence Morinière, Quand la valorisation alimentaire encourage la conservation d'une race : le cas de la race bovine Bretonne Pie Noir, in Annick Audiot, Anne Lauvie, Lucie Markey, Pierre Quéméré, Étienne Verrier (éds), Races en devenir, Ethnozootechnie, 103, 2018, p. 13-18. 
des Alpes du nord qui associent les deux races bovines locales, le lait destiné à ces AOp étant payé aux éleveurs entre $35 \%$ et $60 \%$ au-dessus de la moyenne nationale ${ }^{11},{ }^{12},{ }^{13}$.

Une telle stratégie suppose donc l'existence d'un marché. De ce point de vue, la segmentation des marchés alimentaires, qui est une caractéristique de la France, est un sérieux atout : à défaut d'être des produits de consommation courante, les produits typés ou de qualité supérieure trouvent une bonne part de leurs débouchés dans les repas festifs ou du dimanche. Toutefois, plus le produit a un coût élevé pour les consommateurs, plus vite la limite de quantité globale produite est atteinte, comme cela a pu être observé, par exemple, au début des années 2000 pour le fromage de Beaufort en Savoie ${ }^{14}$.

À une époque où les circuits courts et/ou de proximité sont en vogue, le marché peut être très local, pour des produits plus courants, et donc aux prix plus adaptés à ce type de consommation. Dans ce contexte, on peut s'interroger sur l'impact de l'existence d'une race locale sur les motivations des consommateurs. Des éléments de réponse sont apportés par un sondage réalisé auprès de clients à la ferme ou sur les marchés de produits issus de trois races locales (Tableau 2). Dans les trois cas considérés, la race locale n'apparaît pas comme un élément de motivation premier, contrairement à la qualité conférée aux produits. Pour être plus précis, la notion de race locale n'arrivait jamais dans une première série de réponses spontanées et, ensuite, quand la race était évoquée, $55 \%$ des sondés déclaraient ne pas la connaitre (ou alors juste de nom), $30 \%$ pouvaient en dire quelques mots et $15 \%$ seulement la connaissaient manifestement bien.

11 Étienne Verrier, La place des races bovines Abondance et Tarentaise dans une politique d'aménagement du territoire des Alpes du Nord. II - Une dynamique nouvelle en cours. Bulletin de l'Académie Vétérinaire de France, 68, 1995, p. 193-200.

12 Vincent Chattelier, François Delattre, Evvolution du secteur laitier dans les Alpes du Nord et spécificités des exploitations de cette zone par rapport aux autres massifs montagneux, Rencontres, Recherches Ruminants, 9, 2002, p. 173-176.

13 CNIEL/SIGF, statistiques laitières.

14 Vincent Chatellier, François Delattre, Le prix du lait en Savoie et les filières fromagères AOC Principales évolutions passées et perspectives, Rapport final pour le GIS Alpes-du-Nord, INRA, Nantes, 2006, 63 p. 
Tableau 2 : Motivations des consommateurs en circuit court de produits issus de trois races locales, notées sur une échelle de 0 (très faible) à 5 (très forte). Extrait des résultats du projet CASDAR (20122014) «Varape $»^{15}$.

\begin{tabular}{|c|c|c|c|}
\hline Motivations d'achat & $\begin{array}{c}\text { Bovine } \\
\text { Bretonne Pie Noir }\end{array}$ & $\begin{array}{c}\text { Bovine et race } \\
\text { Rouge Flamande }\end{array}$ & $\begin{array}{c}\text { Caprine } \\
\text { Pyrénéenne }\end{array}$ \\
\cline { 2 - 4 } & 5 & 3 & 4 \\
Qualité des produits & 4 & 0 & 1 \\
Goût typique/unique & 3 & 1 & 3 \\
Échanges avec l'éleveur & 1 & 1 & 2 \\
Produit local & 2 & 0 & 1 \\
Produit Bio & 0 & 0 & 0 \\
Produit de race locale & 0
\end{tabular}

L'existence d'un marché distant mais important, comme peuvent l'être les grands centres urbains, est aussi généralement un atout. À ce titre, le cas de la race bovine Aubrac est très intéressant ${ }^{16}$. Depuis les années 1960, la relance puis le développement de cette race locale, devenue allaitante après avoir été à multiples fins, ont bénéficié de la demande massive en broutards des ateliers d'engraissement de la Plaine du Po, en Italie. Mais ce marché étant ciblé sur un type d'animal précis, à savoir un veau mâle croisé Aubrac x Charolais, les éleveurs ont développé d'autres marchés, avec sIQO, pour les veaux croisés femelles et les animaux de race pure.

\section{Les conséquences du degré d'exigence d'un sIQO et du contrôle par les éleveurs}

L'obtention d'un prix élevé pour les producteurs est-elle une conséquence automatique de la labellisation d'un produit par un sIQO ? Poser la question est déjà un peu y répondre, et l'analyse comparée de différentes situations conduit à répondre clairement par la négative.

Si l'on s'en tient au cas des fromages AOP, la situation très favorable des quatre AOP des Alpes du nord évoquée plus haut s'explique par plusieurs facteurs ${ }^{17},{ }^{18}:(i)$ des cahiers des charges parmi les plus exigeants à tout point de vue parmi les AOP fromagères en France, conférant aux produits une indéniable typicité et un fort pouvoir évocateur ; (ii) la transformation au sein de coopératives de taille modérée ; (iii) un contrôle très étroit de la stratégie produits de la part des éleveurs. On rencontre une situation très comparable pour l'AOP Laguiole, dans le sud du Massif Central, à la différence près que ce fromage est fabriqué et affiné dans une unique coopérative qui lui est dédiée ${ }^{19}$.

15 Pauline Van Ruymbeke, Valorisation en circuits courts de produits issus de 5 races à petits effectifs: visions d'éleveurs, de consommateurs et d'intermédiaires, Mémoire de fin d'études, 2013, 132 p.

16 Jean-Baptiste Borrès et al., 2019, op. cit., p. 107-153.

17 Étienne Verrier, 1995, op. cit.

18 Vincent Chattelier, François Delattre, 2002, op. cit.

19 Jean-Baptiste Borrès et al., 2019, op. cit., p. 156-192. 
Le nord du Massif Central, avec 5 AOp fromagères, montre une situation à l'opposé des cas précédents : (i) des cahiers des charges peu exigeants ; (ii) une fabrication effectuée dans les ateliers de grands groupes coopératifs ou privés ; (iii) des décisions en matière de développement de produits et de prix du lait prises à l'échelle des sièges et non localement. Dans ces conditions, le prix du lait payé aux producteurs est égal à la moyenne nationale, ni plus ni moins.

\section{La nécessaire cohésion des opérateurs}

Les initiatives pour promouvoir les races locales via leurs produits peuvent amener certains opérateurs liés aux produits à s'intéresser aux orientations d'une race donnée, orientations qui étaient historiquement une prérogative des éleveurs. Cela contribue à l'émergence de nouveaux points de vue sur ce qu'est la race et sur les directions vers lesquelles il conviendrait de la faire évoluer. L'analyse du cas de diverses races bovines locales permet de dégager les facteurs qui favorisent la construction d'un consensus et ceux qui, au contraire, conduisent à des controverses ou à des tensions entre acteurs ${ }^{20}$. L'existence de lieux et de temps où les différents points de vue peuvent s'exprimer et être confrontés se révèle critique. C'est une des raisons qui ont poussé le législateur à renforcer, en 2006, la place des représentants des filières de produits et des territoires dans la constitution des organismes de sélection (os), une des missions de l'os d'une race donnée étant d'en fixer les objectifs de sélection (rôle repris depuis 2018 par les os au sens de l'Union Européenne).

Au-delà des structures, il convient de considérer les personnes qui participent aux différents collectifs (collectif race, collectif produit). Le rôle de quelques leaders charismatiques et la force des collectifs se révèlent souvent déterminants ${ }^{21},{ }^{22}$. On peut dire comme une lapalissade que la présence de certains responsables dans les deux collectifs à la fois favorise l'émergence d'un consensus. De ce point de vue, les situations sont contrastées ${ }^{23},{ }^{24},{ }^{25}$. Au titre d'une importante "participation croisée » entre un collectif racial et un collectif AOp, on peut citer les cas de la race bovine Tarentaise et du fromage de Beaufort, de la race bovine Rouge des Prés et du Bœuf Maine-Anjou et de la race porcine Nustrale et de la Charcuterie Corse. Dans ces cas-là, qui se

20 Adeline Lambert-Derkimba, Anne Lauvie, Étienne Verrier, How the development of products valorizing local breeds changes breeding goals: examples from French cattle breeds, Animal Genetic Resources, 53, 2013, p. 135-140.

21 Éric Rousseaux, La puissance du collectif, le cas des races mulassières du Poitou, in Annick Audiot et al., 2018, op. cit., p. 33-39.

22 Jean-Baptiste Borrès et al., 2019, op. cit., p. 500-504.

23 Adeline Lambert-Derkimba, François Casabianca, Étienne Verrier, La place des projets territoriaux dans les prises de décision sur le devenir des races locales, Les Actes du BRG, 7, 2008, p. 509-515.

24 Adeline Lambert-Derkimba, Étienne Verrier, François Casabianca, PDO project for the Corsican pork as an innovation for the whole marketing chain, EAAP Publication Nr 129, 2011, p. 270-277.

25 Adeline Lambert-Derkimba et al., 2013, op. cit. 
rapprochent parfois d'un état fusionnel, on trouve sans surprise une grande cohérence entre les projets raciaux et AOP. À l'opposé, les " atomes crochus » sont rares entre le collectif de l'AOP Beaufort et le collectif de la race bovine Abondance. Cette race locale a beau contribuer pour la moitié à production du lait nécessaire à la fabrication de ce fromage, le collectif AOP se retrouve manifestement plus dans le projet du collectif de la race Tarentaise, et à l'inverse, la production de Beaufort ne représente que $10 \%$ de la valorisation du lait des vaches Abondance, dont le collectif racial se retrouve plus dans les projets des collectifs des autres AOP de la région ${ }^{26}$.

\section{Le nom : un enjeu de communication}

Dans la plupart des cas, une race animale porte le nom de la région dans laquelle un collectif d'éleveurs l'a progressivement constituée (on parle de berceau de race) : race équine Comtoise, race bovine Normande, race ovine Solognote, etc. Dans la même logique, le nom d'un produit bénéficiant d'un siQo provient $\mathrm{du}$ terroir de ce produit : Saint-Nectaire, Crottin de Chavignol, Poulet de Bresse, etc. En plus de désigner une entité, ces noms véhiculent une ou plusieurs images. L'homme étant " un omnivore consommant des végétaux, des animaux et de l'imaginaire ${ }^{27}$, le nom devient un enjeu crucial de communication.

Dans le nord du département de la Haute-Savoie, la commune d'Abondance est célèbre pour son abbaye fondée au XII ${ }^{e}$ siècle et dont le cloître est garni de fresques du XVe . La vallée dans laquelle est nichée cette commune, qui porte le même nom, est le berceau d'une race bovine déjà évoquée ici, tout d'abord désignée comme Chablaisienne puis dénommée officiellement Abondance en $1891^{28}$. Près d'un siècle plus tard, en 1990, le fromage local à pâte pressée cuite a obtenu une AOC (devenue plus tard AOP) sous le nom d'Abondance. Le mot "abondance " étant également un nom commun en français, porteur d'une image franchement positive, le syndicat interprofessionnel du fromage d'Abondance ne manque pas de jouer de cette triple homonymie dans ses campagnes de promotion.

Sans doute consciente du fait que l'homme est un mangeur d'imaginaire (cf. plus haut), l'Union Européenne a édicté en 1992 un règlement qui stipule qu'un même nom géographique ne peut pas être donnée simultanément à un produit bénéficiant d'un indication géographique (IGP ou AOP) et à une race animale. Une loi ne pouvant être rétroactive, le fromage et la race d'Abondance ont gardé leur nom (de même que le fromage et la race bovine de Salers).

26 Adeline Lambert-Derkimba, François Casabianca, Étienne Verrier, L’inscription du type génétique dans les règlements techniques des produits animaux sous AOC: conséquences pour les races animales, INRAE Productions Animales, 19, 2006, p. 357-370.

27 Selon l'expression de Claude Fischler.

28 https://www.races-montagnes.com/fr/races/vache-bovine-abondance.php [consultée le 12 mai 2020]. 
Mais plusieurs collectifs qui n'avaient pas encore obtenu d'appellation pour leur produit avant cette année-là ont été confrontés au problème suivant : il serait opportun d'attribuer au produit le nom géographique porteur d'image, afin de favoriser son écoulement, mais ce nom est déjà celui de la race choisie comme support du produit. D'où le dilemme : doit-on conserver son nom à la race et, pour le produit, choisir un nom moins évocateur, ou bien modifier le nom de la race, malgré des décennies voire plus d'un siècle d'ancienneté, et attribuer le nom évocateur au produit ? La résolution de ce dilemme suppose une bonne entente entre les collectifs de la race et du produit (cf. plus haut). L'expérience montre que, dans tous les cas, la race s'est vue dépossédée de son nom au profit du produit qui lui était associé. C'est ainsi que la race bovine Maine-Anjou est devenue Rouge des Prés, la race bovine Camargue est devenue Raço di Biou (un terme provençal) et la race Porcine Corse est devenue Nustrale (le nôtre en corse).

Les produits à forte valeur ajoutée ou au débouché local bien établi peuvent contribuer à préserver, voire développer, des races locales qui furent un temps délaissées mais peuvent trouver une « deuxième jeunesse » à la faveur des évolutions des comportements des citoyens-consommateurs que nous sommes. De façon réciproque, une race locale peut donner une image positive à un produit (même si des enquêtes auprès des consommateurs tendent à relativiser l'importance de ce phénomène), ou tout du moins l'aider à se démarquer du tout-venant. Il s'agit donc d'une association à bénéfices réciproques.

Les nombreuses démarches de valorisation de races locales qui se sont développées au cours des dernières décennies ont montré qu'il n'existe aucune " recette miracle " et qu'il faut faire preuve à la fois d'imagination et de réalisme face aux opportunités et contraintes du lieu et du moment. En particulier, l'obtention d'un label officiel ne suffit pas à tirer les prix vers le haut. Le contrôle des filières par les éleveurs et la cohésion des collectifs liés à la race et au produit demeurent essentiels. Enfin, les produits étant à destination d'une population qui se pose de plus en plus de questions quant à ses sources d'approvisionnement alimentaire, il est important que les enjeux relatifs à la biodiversité domestique soient mieux connus du grand public.

Étienne Verrier

AgroParisTech, UMR GABI (Inrae/AgroParisTech) etienne.verrier@agroparistech.fr

Lucie Markey Institut de l'Élevage. Coordination du projet Casdar « Varape », 2012-2014 lucie.Markey@idele.fr

Anne Lauvie

INRAE. Coordination du projet « Secoya » anne.lauvie@inrae.fr 
Étienne Verrier est professeur de génétique animale à AgroParisTech et directeur-adjoint de l'UMR GABI (Inrae/AgroParisTech). Ses travaux portent sur la définition d'indicateurs pour la gestion génétique des populations animales et la valorisation et l'inscription territoriale des races locales.

Lucie Markey est chef de projet à l'Institut de l'Élevage. Ses missions concernent la gestion des ressources génétiques, et l'appui au développement de démarches de valorisation des produits des races à petits effectifs (coordination du projet Casdar «Varape » en 2012-2014).

Anne Lauvie est chercheur à l'INRAE. Ses travaux portent sur la gestion territoriale des populations animales locales. En 2017-2018, elle a coordonné le projet "Secoya » qui visait à rendre compte de la diversité des contributions des élevages qui utilisent des races locales.

\title{
Résumé
}

Sur la base de cas concrets relatifs à plusieurs espèces domestiques, en France, on tire cinq enseignements relatifs à la valorisation des races locales via leurs produits. Premièrement, il n'existe pas de solution unique pour valoriser une race, une grande diversité des moyens se rencontrant et parfois se combinant sur le terrain. Deuxièmement, il est nécessaire de construire et de maintenir une clientèle et d'adapter en temps réel la production et demande. Troisièmement, un défi de taille est de maintenir un contrôle étroit des éleveurs sur la filière, de sorte que la valeur ajoutée leur bénéficie pleinement. Quatrièmement, il convient de veiller à la cohésion entre les différents acteurs et de porter une attention équilibrée au développement des produits et à la gestion des races. Cinquièmement, les collectifs concernés doivent gérer avec habileté la question de l'appropriation, par la race ou par le produit, du nom porteur d'une image favorable auprès du public.

\section{Mots-clés}

Biodiversité domestique, races locales, territoires, produits.

\begin{abstract}
Added value for local breeds by products linked to their territory: five lessons learned from various cases in France. On the basis of concrete cases from several domestic species, in France, five lessons were drawn on how to obtain an added value to local breeds via their products. First, there is no single way to find an added value, a wide variety of means being encountered and sometimes combined on the field. Second, it is necessary to build and maintain a customer base and to adapt production and demand in real time. Third, a major challenge is to maintain a close control of farmers on the value chain, so that the added value benefits them fully. Fourth, there is a need to ensure cohesion between the different actors and to pay attention to the balance between product development and breed management. Fifth, the groups of concerned people must skillfully manage the issue of the appropriation of the name carrying a favorable image, by the breed or by product.
\end{abstract}

\section{Keywords}

Domestic biodiversity, local breeds, territories, products. 